\title{
Measurement of the Loss and Depolarization Probability of UCN on Beryllium and Diamond Like Carbon Films
}

Volume 110

\section{Tomas Brys}

Paul Scherrer Institut, 5232 Villingen PSI,

Switzerland

and

ETH Zürich,

Zurich, Switzerland

\section{Manfred Daum}

Paul Scherrer Institut, 5232 Villingen PSI,

Switzerland

\section{Peter Fierlinger}

Paul Scherrer Institut, 5232 Villingen PSI, Switzerland

and

Universitaet Zürich,

Zurich, Switzerland

\section{Peter Geltenbort}

Institut Laue Lagevin, Grenoble, France

\section{Mukul Gupta}

Paul Scherrer Institut, 5232 Villingen PSI,

Switzerland

Reinhold Henneck

Paul Scherrer Institut, 5232 Villingen PSI,

Switzerland

\section{Stefan Heule}

Paul Scherrer Institut, 5232 Villingen PSI, Switzerland

and

Universitaet Zürich, Zurich, Switzerland

\section{Klaus Kirch}

Paul Scherrer Institut, 5232 Villingen PSI, Switzerland
Number 3

May-June 2005

\author{
Mikhail Lasakov \\ PNPI Gatchina, Russia \\ Russel Mammei \\ Virginia Tech, \\ Blacksburg, USA \\ Mark Makela \\ Virginia Tech, \\ Blacksburg, USA
}

\section{Axel Pichlmaier}

Paul Scherrer Institut, 5232 Villingen PSI,

Switzerland

Anatoli Serebrov

Paul Scherrer Institut,

5232 Villingen PSI,

Switzerland

and

PNPI Gatchina, Russia

\section{Ulrich Straumann}

Universitaet Zürich,

Zurich, Switzerland

Robert B. Vogelaar

Virginia Tech,

Blacksburg, USA

\section{Cedric Wermelinger}

ETH Zürich,

Zurich, Switzerland

and

Paul Scherrer Institut,

5232 Villingen PSI,

Switzerland

and

Albert Young

North Carolina State University,

Raleigh, USA

peter.fierlinger@psi.ch axel.pichlmaier@psi.ch

\begin{abstract}
Currently several institutes worldwide are working on the development of a new generation of ultracold neutron (UCN) sources. In parallel with source development, new materials for guiding and storage of UCN are developed. Currently the best results have been achieved using ${ }^{58} \mathrm{Ni}, \mathrm{Be}$, solid $\mathrm{O}_{2}$ and low temperature Fomblin oil (LTF). All of these materials have their shortcomings like cost, toxicity or difficulty of use. A novel very promising material is diamond like carbon (DLC). Several techniques exist to coat surfaces, and industrial applications (e.g., for extremely hard surfaces) are already wide spread. Preliminary investigations using neutron reflectometry at PSI and Los Alamos yielded a critical velocity for DLC of about $7 \mathrm{~m} / \mathrm{s}$ thus comparable to Beryllium. A low upper limit of depolarization probability for stored polarized $\mathrm{UCN}$ has been measured at the PF2 facility of the Institut Laue-Langevin (ILL) by North Carolina State University (NCSU), Los Alamos National Laboratory (LANL), and Petersburg Nuclear Physics Institute (PNPI), thus making it also a good material for storage and guidance of polarized UCN. Still missing is the loss probability per bounce. We will be able to extract this number and a more stringent value for the depolarization from our experiment thus proving the suitability of DLC as a wall material for a wide range of UCN applications.
\end{abstract}

Key words: depolarization; diamond like carbon; spin flip; ultracold neutrons.

Accepted: August 11, 2004

Available online: http://www.nist.gov/jres

\section{Introduction}

Currently, strong efforts are under way worldwide to build new sources for ultracold neutrons (UCN)(see e.g., [1], [2], [3]). All of these new sources will use solid deuterium $\left(\mathrm{sD}_{2}\right)$ as neutron moderator. Hand-inhand with the development of these sources, new materials to store and guide the $\mathrm{UCN}$ are also required. 
The material of choice is Beryllium since it has a high critical velocity of $6.9 \mathrm{~m} / \mathrm{s}$, equal to Nickel and second to isotopically enriched ${ }^{58} \mathrm{Ni}\left(v_{\text {crit }}=8.1 \mathrm{~m} / \mathrm{s}\right)$. Nickel however is magnetic and is not suitable for polarized UCN. Beryllium on the other hand is toxic and therefore difficult to machine and handle. One promising alternative material is diamond-like carbon (DLC) which is non-toxic, hydrophobic, stable, and widely used in industrial applications. Existing technology can be used allowing a cost effective production. Experience with DLC already exists [4], [5]. At SINQ we have recently measured the critical velocity of DLC and found it to be $(7.0 \times 0.3) \mathrm{m} / \mathrm{s}$, similar to Beryllium. The quality of the coatings was further investigated by thermal cycling between $450 \mathrm{~K}$ and $77 \mathrm{~K}$ and gave stable results; the adhesion to the substrate turned out to be excellent. Contamination with other elements, especially Boron, is a concern but depends directly on the quality of the raw material used. It is small and well under control.

Scientifically most interesting is a measurement of the depolarization probability per bounce. It has been investigated in earlier experiments for DLC [5] and for Be [6] during storage. It was found to be below $3 \times 10^{-6}$ per bounce for DLC and around $2 \times 10^{-5}$ to $3 \times 10^{-5}$ for Beryllium. A more detailed investigation of the spin flip probability as a function of energy and temperature, which we propose here, is important in order to

- clarify whether there is indeed a connection between spin flip and "anomalous losses," as has been proposed recently ([6], [7]). In this picture, some UCN are spin flipped upon reflection due to incoherent scattering from para/ferro-magnetic centers on the material surface. The fraction of UCN scattered incoherently into the material could possibly explain the observed "anomalous losses." In order to study this experimentally, one has to measure both parameters, the depolarization and the loss probability per wall collision, simultaneously in the same experiment;

- to supply information on the polarization stability during storage. Precision UCN experiments rely on the fact that the change of polarization during storage does not introduce systematic errors. Obviously, improving the accuracy for the planned experiments also places stronger constraints on the control of the polarization. Although the sensitivity to depolarization effects can be inherently low, depolarization aspects need to be studied for future high-sensitivity studies [2].
Contrary to the other depolarization experiments our set-up has no mechanical slits. Although other experiments typically found wall losses in the order of $5 \times 10^{-5}$ per bounce, theory predicts values two orders of magnitude lower [8]. The experiment thus allows to pin down both, the loss and the depolarization probability per bounce.

We use a vertical storage tube sealed on the top by gravity and on the bottom by a conventional $1.5 \mathrm{~T}$ magnet, corresponding to a neutron energy of $90 \mathrm{neV}$. The neutrons have no contact to any material surface other than the coating (DLC or Be). Since earlier experiments have been crippled by poor vacuum conditions, special care is taken to achieve the best possible vacuum. We use well established vacuum techniques like baking and usage of a cryo cooler for hydrogen pumping. We are able to adjust the temperature between $40 \mathrm{~K}$ and $450 \mathrm{~K}$.

\section{Experimental Set up and Expected Results}

The experiment is carried out at the ILL-PF2 ultra cold neutron installation. It works as follows: UCN from the turbine enter the storage volume (1, see Fig. 1) through the feeding guide (4), an Aluminum window and a beam switch (3). At this point the magnet (2) is still switched off, but the $10^{-2} \mathrm{~T}(100 \mathrm{G})$ holding field (6) in the storage volume is already on. After equilibrium neutron density is reached, the magnet is turned on. This can be done in about $3 \mathrm{~s}$. Now the beam switch is moved such that the storage volume is connected to the detector (5). Neutrons with the "wrong" spin state drain quickly from the storage volume while the others are confined by the magnetic field, the coated walls and gravity (see Fig. 2). After a certain holding time the magnet is switched off and the stored neutrons fall into the detector. This procedure is repeated with two different holding times $\left(t_{1}, t_{2}\right)$ with the number of stored neutrons $N_{t 1}$ and $N_{t 2}$, respectively, thus yielding the neutron lifetime in the storage volume $\tau_{\text {tot }}$ Eq. (1):

$$
\tau_{\text {tot }}=\frac{t_{1}-t_{2}}{\ln \left(N_{t 1} / N_{t 2}\right)} .
$$

Using $\tau_{\text {tot }}$ and the total number of wall collisions $v$ determined by Monte Carlo simulations, the loss probability $\mu$ per wall interaction can be calculated Eq. (2).

$$
\frac{1}{\tau_{\mathrm{tot}}}=\frac{1}{\tau_{\mathrm{n}}}+v \beta+v \mu \text {. }
$$




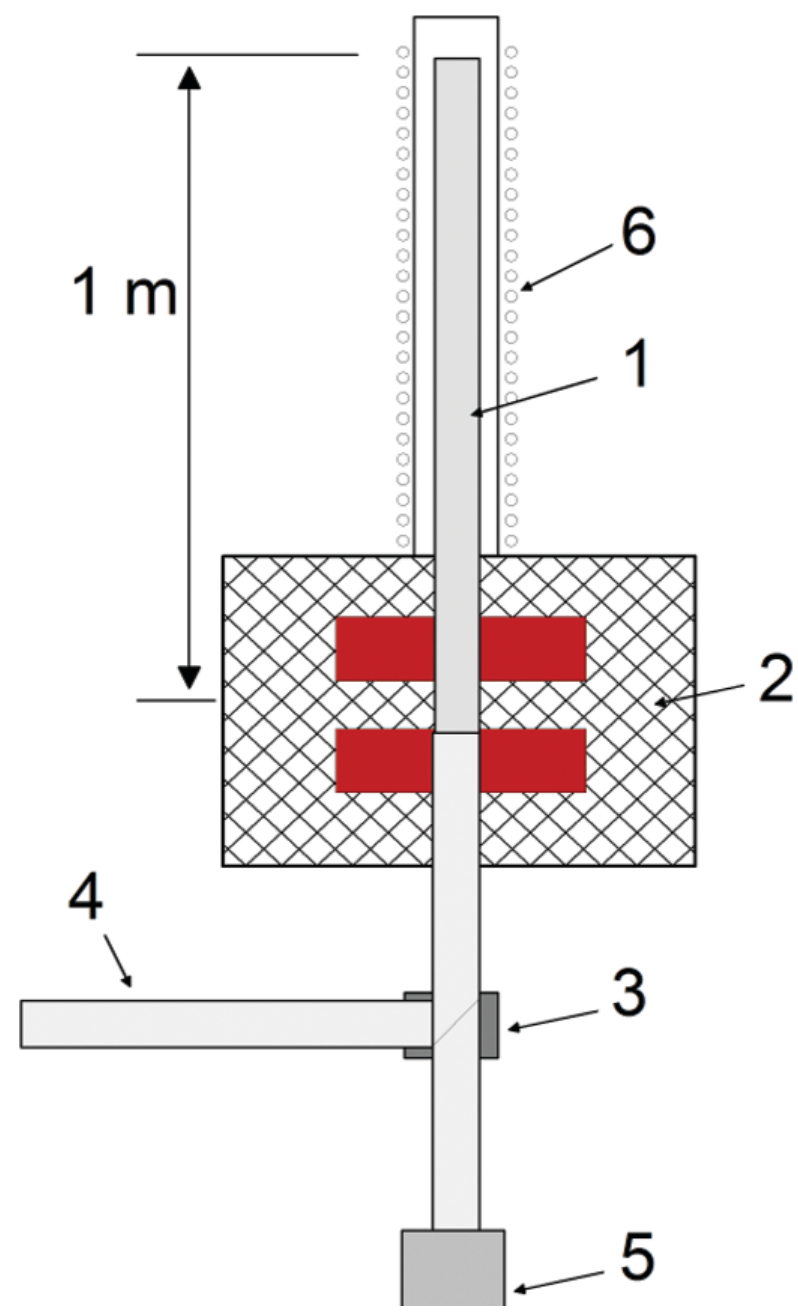

Fig. 1. Set up of the experiment. 1 - sample tube, 2 - magnet, 3 - beam switch, 4 - feeding guide, 5- UCN detector, 6 - holding field coil.

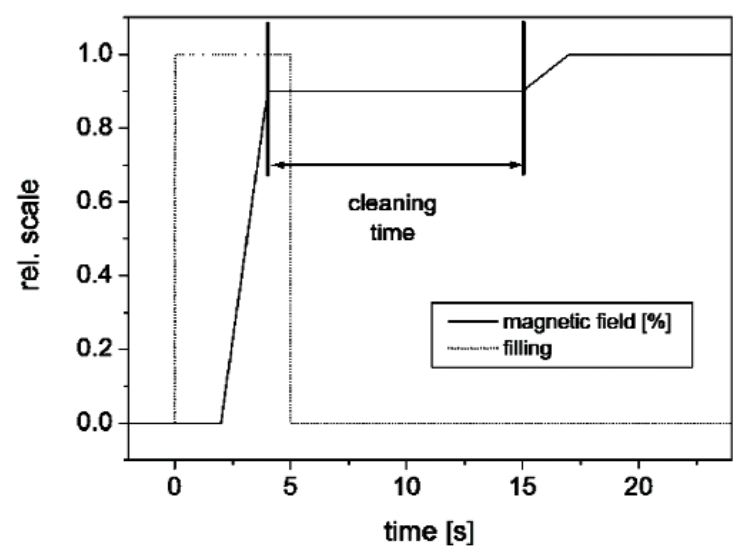

Fig. 2. Ramping of the magnetic field during filling and spectral cleaning. The field is ramped up to $90 \%$ of the maximum value while the neutrons are filled into the storage volume. Neutrons with "wrong" spin state or too high energy are effectively removed during the cleaning time. The magnet is then ramped to maximum field and the storage measurement starts.
Neutrons that experience a spin flip $\left(\mathrm{N}_{\mathrm{sp}}\right)$ during the storage are no longer confined by the magnetic field. They are counted in the detector during the holding time. The spinflip probability $\beta$ can be calculated according to Eq. (3).

$$
N_{\mathrm{sp}}=\left(N_{t 1}-N_{t 2}\right) \cdot \tau_{\mathrm{tot}} \cdot v \cdot \beta .
$$

Since the background count rate is in the order of only a few $\mathrm{ms}^{-1}$, and since the "wrong" spin state drains within seconds from the storage volume, we detect a clear signal from the depolarized neutrons.

Alternatively we fill the storage volume with the magnet on and store only depolarized UCN. This serves as a complementary measurement cycle which helps to exclude systematic uncertainties. It must give the same result if all parameters are well under control.

With a loss factor $\mu=10^{-4}$ and a bottle lifetime of $180 \mathrm{~s}$, filling and emptying time of around $2 \mathrm{~s}$ can be expected. This enables us to get $\mathrm{a} \cong 15 \%$ statistical accuracy for the storage time $\tau$ and $\cong 20 \%$ for the spin flip probability $\beta$ at $\beta=3 \times 10^{-6}$ in one measurement cycle of about $15 \mathrm{~min}$.

We will test DLC coatings made by two different techniques on Quartz and Aluminum substrates. For reference we will also measure Beryllium coated surfaces.

\section{Acknowledgments}

We would like to thank Karel Kohlik and the PSI workshops for their competent and fast help building the experiment. At the ILL we like to thank Thomas Brenner for the local support.

\section{References}

[1] http://www.lanl.gov/orgs/p/progrpt 99 00/research/nuc_ucn.pdf (accessed September 22, 2003).

[2] http://ucn.web.psi.ch/ (accessed May 24, 2004).

[3] http://www.frm2.tu-muenchen.de/frm2/secsources/de/ucn.html (accessed May 24, 2004).

[4] M. G. D. van der Grinten et al., Nucl. Instr. and Meth. A423, 421 (1999).

[5] Proposal to ILL experiment 3-14-150 and 3-14-163.

[6] A. Serebrov et al., Nucl. Instr. and Meth. A440, 717 (2000).

[7] A. Serebrov et al., Phys. Lett. A. 313, 373-379 (2003).

[8] R. Golub and J. M. Pendlebury, Rep. Prog. Phys. 42, 439 (1979). 